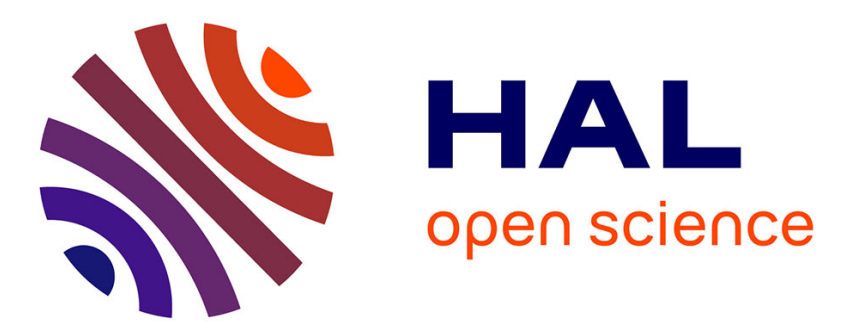

\title{
On the Use of Fixed-Intensity Functional Electrical Stimulation for Attenuating Essential Tremor
} Antonio Padilha Lanari Bo, Christine Azevedo Coste, Christian Geny, Philippe Poignet, Charles Fattal

\section{- To cite this version:}

Antonio Padilha Lanari Bo, Christine Azevedo Coste, Christian Geny, Philippe Poignet, Charles Fattal. On the Use of Fixed-Intensity Functional Electrical Stimulation for Attenuating Essential Tremor. Artificial Organs, 2014, 38 (11), pp.984-991. 10.1111/aor.12261 . lirmm-00951194

\section{HAL Id: lirmm-00951194 https://hal-lirmm.ccsd.cnrs.fr/lirmm-00951194}

Submitted on 7 Mar 2014

HAL is a multi-disciplinary open access archive for the deposit and dissemination of scientific research documents, whether they are published or not. The documents may come from teaching and research institutions in France or abroad, or from public or private research centers.
L'archive ouverte pluridisciplinaire HAL, est destinée au dépôt et à la diffusion de documents scientifiques de niveau recherche, publiés ou non, émanant des établissements d'enseignement et de recherche français ou étrangers, des laboratoires publics ou privés. 


\title{
On the use of fixed-intensity FES for attenuating Essential Tremor
}

Antônio Padilha Lanari Bó ${ }^{1}$, Christine Azevedo-Coste ${ }^{2}$, Christian Geny ${ }^{3}$, Philippe Poignet ${ }^{4}$, Charles Fattal $^{5}$

\author{
${ }^{1}$ LIRMM/Neuromedics, France \\ Current address:
}

Dept. Engenharia Eletrica, Universidade de Brasilia, Campus Universitario Darcy Ribeiro, CEP:70910-900, Brasilia, Brazil Tel.: +55-61-31071040

Fax: +55-61-31075590 antonio.plb@lara.unb.br

${ }^{2}$ LIRMM/INRIA Sophia-Antipolis, France

${ }^{3}$ Centre Hospitalier Universitaire de Montpellier, France

${ }^{4}$ LIRMM/Université Montpellier 2, France

${ }^{5}$ Centre Mutualiste Neurologique Propara, France

\begin{abstract}
Traditional therapies have not been able so far to provide functional benefits for a great part of Essential Tremor (ET) patients. In this scenario, the use of Functional Electrical Stimulation (FES) has been proposed for reducing tremor amplitude by stimulating muscles in anti-phase with respect to trembling motion. Although some studies have reported success in terms of tremor attenuation, drawbacks still exist which prevent using the method in real-life applications. In this paper, we explore an alternative approach: a strategy based on the hypothesis that FES-induced constant muscle contraction may provide a functional benefit for tremor patients. To evaluate the proposed strategy, experiments were conducted in which stimulation was intermittently turned on and off while the subjects performed a static motor task. The results of the proposed experimental protocol have indicated that tremor attenuation using this strategy is feasible, since consistent tremor attenuation levels were obtained in 8 out of 10 ET patients. Nonetheless, tremor reduction was not instantaneous for all successful trials, indicating that prior training with FES may improve the overall response. Furthermore, although simpler assistive devices may be potentially designed based on this technique, some experimental difficulties still exist, which suggest further studies are necessary.
\end{abstract}

Keywords: Essential Tremor, Functional Electrical Stimulation, Fixed-intensity stimulation 


\section{INTRODUCTION}

Tremor, which concerns involuntary, approximately periodic, and roughly sinusoidal movement, is one of the most common movement disorders [Lyons and Pahwa, 2005]. In particular, Essential Tremor (ET) is a monosymptomatic pathology whose patients usually present postural tremor aggravated by voluntary movement, which thus considerably decreases their ability to perform simple daily tasks. Currently, the main available treatments for ET are based on drugs and invasive interventions, such as Deep Brain Stimulation (DBS). While the first lacks effectiveness, since limited positive outcome is obtained for many patients, the second still presents high cost and possibly produces side effects [Grimaldi and Manto, 2008].

In order to enable the development of new therapies, a suitable approach would be to investigate pathological tremor inner dynamics, such as in [Zhang et al., 2009], including the relation between central oscillators and peripheral mechanisms in the origin of ET. However, to our knowledge no results have been obtained using this methodology so far, and hence alternative treatments based on the use of assistive technology have been proposed. One example of such devices is the active upper limb exoskeleton [Rocon et al., 2007], an orthosis that is directly coupled to the joints and actuated in order to counteract tremor. The referred system presented satisfactory performance in preliminary trials; nevertheless its unwieldiness avoids practical use in daily life.

In order to directly actuate on the concerning trembling joints, an option would be to apply Functional Electrical Stimulation (FES). FES is based on the principle of delivering electric pulses to the muscle to produce contraction, either to move the limb or modulate the joint impedance by co-contraction [Popovic and Sinkjaer, 2000]. Comparing to active orthoses, FES systems are potentially lighter and smaller, and there is no need of adjusting the device for different users. However, in spite of its potential for different applications, practical use of FES still presents significant challenges [Lynch and Popovic, 2008], particularly when superficial electrodes are used.

From an engineering perspective, such FES systems must contain sensors that detect or estimate the time-varying tremor features. Based on this information, stimulation parameters may be modulated in real-time to reduce tremor, while minimizing interference in voluntary motion. In [Bó et al., 2011], we have targeted the problem of tremor estimation in real-time. The current paper concerns the study of adequate FES actuation strategies to attenuate the effects of pathological tremor.

Pioneer studies in the topic [Prochazka et al., 1992] and also more recent studies, such as [Maneski et al., 2011] and [Zhang et al., 2011], were based on applying FES to activate antagonist muscles in anti-phase with respect to trembling motion in order to reduce tremor amplitude. Using this strategy, it has been demonstrated experimentally that tremor attenuation using FES is feasible. Moreover, in some cases the positive effect was instantaneous and enduring. 
Nevertheless, drawbacks of the method have come to light that still prevent current use in daily life by patients. First, these methods rely on FES-induced motion, which is a great obstacle for daily use due to difficulties concerning electrode placement and because muscle activation from surface electrodes change during forearm motion (i.e., for different forearm orientations, the same electrodes may be stimulating different muscle groups). Also, increase in tremor amplitude may occur due to errors in tremor phase estimation or in controller parameters, which may be aggravated if manual tuning of parameters before each use is required, as in [Prochazka et al., 1992]. Lastly, greater discomfort caused by time-varying stimulation intensity was felt by some patients.

An alternative to anti-phase stimulation is based on the idea of increasing the affected joint impedance using electrical stimulation and, in consequence of that, reducing tremor amplitude. The approach is inspired by strategies often employed by tremor patients in daily life, such as co-activating the muscles to amplify joint impedance and supporting the trembling limb against a fixed object, such as a table, or holding it with the unaffected hand. In terms of musculoskeletal dynamics, increasing joint impedance without producing any residual joint motion is possible when antagonist muscles deliver the same, but opposing torques to the joint. In this condition, joint impedance may be modulated by the muscles activation level due to both intrinsic and proprioceptive contributions to muscle active viscoelasticity [Winters and Crago, 2000].

Preliminary evaluation of this approach indicating good results has already been performed both in simulation [Bó et al., 2009], on healthy subjects [Bó and Poignet, 2010], and tremor patients [Gallego et al., 2013]. It is a promising approach, since the applied stimulation might be more comfortable and produce less fatigue, due to the constant lowlevel stimulation applied. Furthermore, the resulting portable device may be simpler, which is an important advantage considering that tremor often propagates from proximal to distal joints. Nonetheless, in the referred works, a closedloop system that modulates the FES level based on the estimated tremor intensity is used. For that reason, time-varying stimulation due to the closed-loop system dynamics is applied, possibly preventing precise understanding and, more important, quantification of which phenomenon produced actual tremor attenuation.

In view of this situation, in this paper we propose a new experimental protocol for evaluating FES tremor attenuation in which fixed-intensity FES is applied on muscles acting on the trembling joint while a static task is performed. By maintaining the maximum number of variables fixed, we expect to attain accurate information on how this complex pathological motion may be compensated. Also, since the ultimate goal is not to completely suppress tremor, but instead to provide the greatest functional benefit, the proposed scheme may present the additional advantage of enabling the subject to easily adapt his/her motion patterns, since FES intensity is constant. 
In order to perform a preliminary evaluation of the method, experiments with 10 ET patients were conducted. The trials featured an initial tremor characterization, where the stimulated muscles were chosen, and subsequent static tests using an open-loop FES system. Since the protocol was based on single-session experiments, patients could not provide an evaluation of functional benefit obtained in daily use. However, tremor amplitude attenuation could be quantified based on the obtained data and the feasibility of the approach was demonstrated. Furthermore, the obtained data illustrated heterogeneous phenomena, hence in this paper we are also focused on discussing their possible causes and potential advantages comparing to alternative methods.

\section{MATERIALS AND METHOD}

Participants - A group of 10 ET patients participated in the study. In Tab. 1, information concerning the participants is listed. The subjects were selected randomly from patients at the Centre Hospitalier Universitaire de Montpellier and they presented heterogeneous tremor features, both in terms of age of tremor onset and severity according to the Fahn-Tolosa-Marin tremor rating scale. None of the patients had experienced surface electrical stimulation beforehand. The study was conducted in accordance with the Declaration of Helsinki and all participants have signed informed consent, which was approved by the local ethical committee.

Table 1: Descriptive data of all patients that participated in the study.

\begin{tabular}{|c|c|c|c|c|}
\hline Pat. & Gender & Age & Age at onset & $\begin{array}{c}\text { Postural tremor at the } \\
\text { dominant arm (Fahn- } \\
\text { Tolosa-Marin tremor } \\
\text { rating scale }{ }^{\mathrm{a}}\end{array}$ \\
\hline \hline A & M & 54 & 10 & 2 \\
B & F & 78 & 15 & 3 \\
C & M & 71 & 56 & 2 \\
D & M & 57 & 6 & 3 \\
E & M & 77 & 10 & 2 \\
F & M & 75 & 73 & 3 \\
G & M & 76 & 32 & 4 \\
H & M & 71 & 1 & 2 \\
I & M & 79 & 20 & 4 \\
J & F & 65 & 60 & 2 \\
a More severe tremors are represented by higher values of the Fahn-Tolosa-Marin tremor \\
scale.
\end{tabular}

Design - The study was based in a within-subject design, which was designed to evaluate the feasibility of the proposed method. The experiments were limited to a single session, where participants were given a static motor task to perform. The relative tremor amplitude was measured during alternating periods when fixed-intensity FES applied to the wrist or fingers was turned on and off. 
Materials - The experimental setup consisted of a commercial stimulator, the Cefar Physio 4 (Cefar, Sweden), and motion sensing units composed of 3-axis accelerometer (MMA7260Q, Freescale, USA) and 2-axis gyrometer (IDG300, Invensense, United States) sampled at $100 \mathrm{~Hz}$. The sensor box was placed using a fastening tape either on the fingers or on the hand, depending on the trembling joint. Within our setup, $x$ axis was pointing distally, $y$ axis medially, and $z$ axis anteriorly. Self-adhesive round $3.2 \mathrm{~cm}$ electrodes were used in the experiment. For smaller muscles in the forearm, the electrodes were downsized to provide better selectivity. Since the stimulation intensity was set manually, we have also used electromyography (Biovision, Germany) coupled with the inertial sensors acquisition system. Based on the corresponding stimulation artifact, we could guarantee precise synchronization between FES and motion signals recorded using inertial sensors.

Procedure - Within the designed experimental protocol, firstly the pathological motion was analyzed using inertial sensors in order to designate the appropriate target joint(s) for stimulation (wrist, fingers, or thumb/index), as well as the concerned muscles. The joint presenting higher tremor amplitude, considering that tremor propagates from proximal to distal joints, was selected. Once these definitions were made, FES parameters were set for each muscle individually while the participants were performing a static motor task: to point to a target with the hand, maintaining the arm in full extension. Furthermore, the limb was not constrained in any possible degree of freedom (DOF). FES pulse width was fixed at $150 \mu s$, and frequency at $40 \mathrm{~Hz}$, while stimulation level was regulated manually using pulse amplitude ( $m A$ ) based on visual and tactile inspection of muscle contraction, but respecting patient subjective evaluation of discomfort. The main goal for setting the stimulation parameters was to produce consistent isometric contraction, while avoiding reaching the motion threshold. Since multiple muscles may affect joint motion, within the initial setup different candidate muscles were stimulated, and the muscle(s) that presented most effective response based on visual inspection was selected for evaluation.

Following the initial setup, evaluation was based on the application of constant stimulation levels intermittently. Stimulation duration was incremented progressively from approximately 10 up to $50 s$ to enable evaluation of different effects that may affect tremor attenuation. Periods in which FES was turned off lasted for at least $10 \mathrm{~s}$, while longer intervals were employed when subjects were not able to reestablish static hand position immediately after stimulation. A sequence ranging from 5 to 7 stimulation periods were applied during each session. Before the stimulation trials started, the patients initiated the static task and remained in this position for at least $10 \mathrm{~s}$ in order to minimize transient effects. If requested by the subject, resting periods were allowed between stimulation periods to limit overall muscle fatigue. Furthermore, it was not possible in any stimulation trial to start stimulation directly at the assigned intensity. Instead, due to the limitations of the employed stimulator, current amplitude was increased until the target level was achieved, in 
a procedure that took approximately $5 s$ to complete. During the whole session, upper limb motion was measured using accelerometers and gyrometers.

Afterward, the resultant recorded motion was analyzed to enable comparison of tremor amplitude with and without FES and identify other emerged effects. The data used in the analysis were preprocessed (high-pass 10th-order Butterworth filter, cutoff frequency at $1 \mathrm{~Hz}$ ) to remove low frequency components. Considering the tasks performed are static, the filtering was applied mainly to remove sensor bias from the signal.

\section{RESULTS}

The results obtained in the study are summarized in Tab. 2 and Figs. 1 and 2. In addition to the quantitative data related to tremor attenuation experiments, Tab. 2 also includes information concerning the target joint and the stimulated muscles, as well as a classification of the overall response to FES therapy in 3 classes: no positive effect, attenuation preceded by an adaptation phase and clear immediate attenuation. Tremor amplitude was computed using the Root Mean Square (RMS) value of the referred motion signal, while tremor attenuation is defined by the ratio

$$
\frac{R M S_{\text {off }}-R M S_{\text {on }}}{R M S_{\text {off }}}
$$

where on and off concerns all periods in which stimulation was on and off, respectively. The results concerning the overall response to FES were obtained directly by analyzing the acquired motion signals, particularly the transient response when FES was turned on and off.

Concerning Tab. 2, the results illustrate the overall success of the method, particularly since in only 1 out of 10 patients the trials resulted in increasing tremor amplitude and in 1 out of 9 patients tremor attenuation was lower than $37 \%$. 
Table 2: Data resulting from the experiments.

\begin{tabular}{|c|c|c|c|c|}
\hline Pat. & Target joint & $\begin{array}{l}\text { Stimulated } \\
\text { muscle }(\mathrm{s})^{\mathrm{a}}\end{array}$ & $\begin{array}{l}\text { Most significant tremor } \\
\text { attenuation }[\%]^{\mathrm{b}} \\
\end{array}$ & Overall response \\
\hline $\begin{array}{l}\mathrm{A} \\
\mathrm{B}\end{array}$ & $\begin{array}{l}\text { Fingers } \\
\text { Wrist }\end{array}$ & $\begin{array}{c}\text { FDS } \\
\text { FCU, ECU }\end{array}$ & $\begin{array}{r}-65.49 \\
12.53\end{array}$ & No positive effect \\
\hline $\begin{array}{l}\mathrm{C} \\
\mathrm{D} \\
\mathrm{E}\end{array}$ & $\begin{array}{c}\text { Fingers } \\
\text { Fingers } \\
\text { Thumb/index }\end{array}$ & $\begin{array}{c}\text { EDC, FDS, PT } \\
\text { FCR } \\
\text { AP, APB }\end{array}$ & $\begin{array}{l}94.68 \\
78.20 \\
72.07\end{array}$ & $\begin{array}{c}\text { Attenuation preceded } \\
\text { by an adaptation } \\
\text { phase }\end{array}$ \\
\hline $\begin{array}{c}\mathrm{F} \\
\mathrm{G} \\
\mathrm{H} \\
\mathrm{I} \\
\mathrm{J}\end{array}$ & $\begin{array}{c}\text { Wrist } \\
\text { Wrist } \\
\text { Wrist } \\
\text { Fingers } \\
\text { Thumb/index }\end{array}$ & $\begin{array}{c}\text { ECU, FCU } \\
\text { PT } \\
\text { EDC } \\
\text { EDC } \\
\text { AP, APB }\end{array}$ & $\begin{array}{l}85.69 \\
41.73 \\
37.18 \\
47.28 \\
78.19\end{array}$ & $\begin{array}{l}\text { Clear immediate } \\
\text { attenuation }\end{array}$ \\
\hline
\end{tabular}

${ }^{\mathrm{a}}$ FDS stands for flexor digitorum superficialis, FCU for flexor carpi ulnaris, ECU for extensor carpi ulnaris, EDC for extensor digitorum communis, PT for pronator teres, FCR for flexor carpi radialis, AP for adductor pollicis, APB for abductor pollicis brevis.

${ }^{\mathrm{b}}$ Most significant tremor attenuation concerns reduction in tremor amplitude on the sensor axis presenting higher tremor intensity before stimulation. For patient A, the minus sign indicates that the performance was worse when FES was on. 


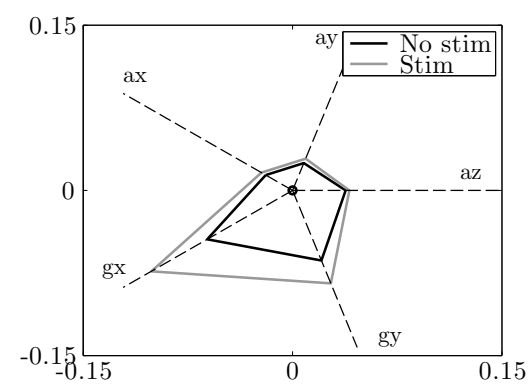

(a) Patient $\mathrm{A}$

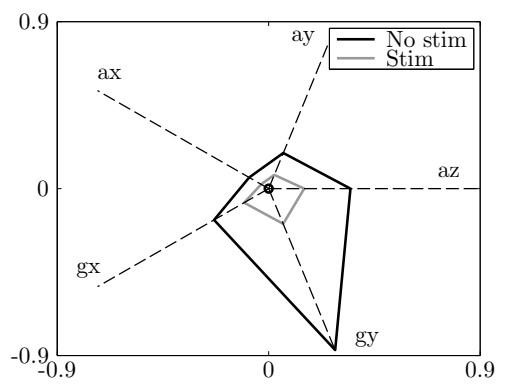

(d) Patient D

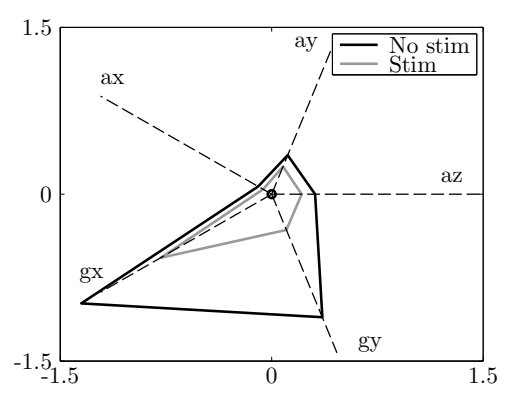

(g) Patient G

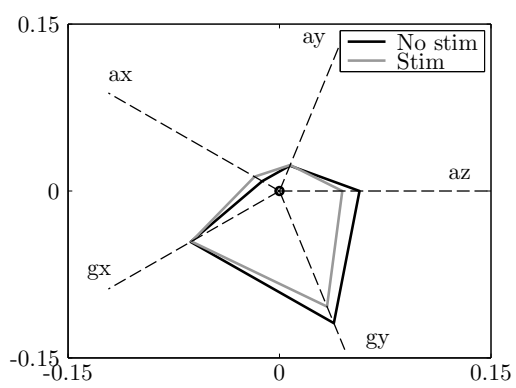

(b) Patient B

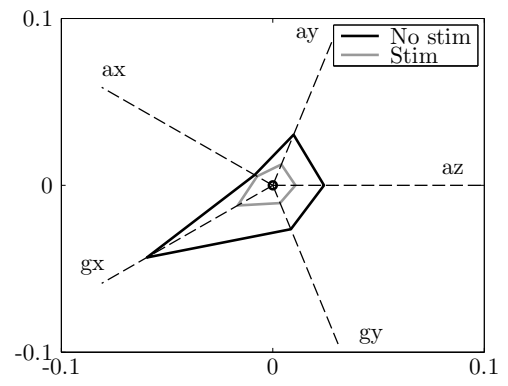

(e) Patient E

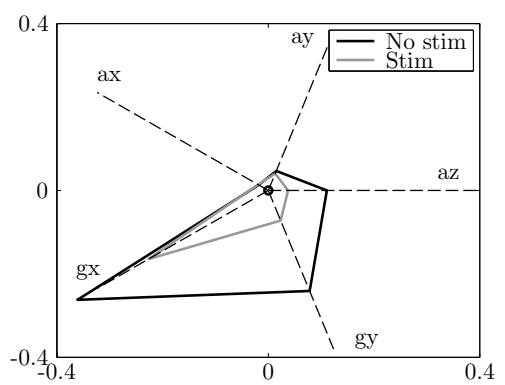

(h) Patient $\mathrm{H}$

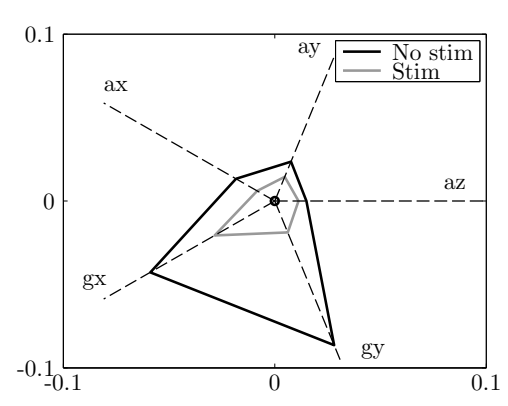

(j) Patient J

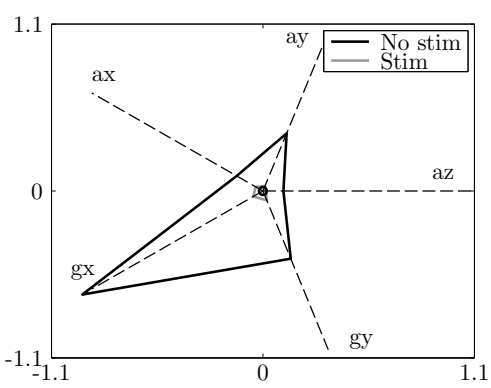

(c) Patient $\mathrm{C}$

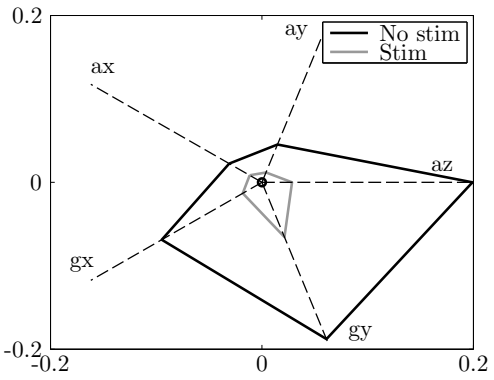

(f) Patient F

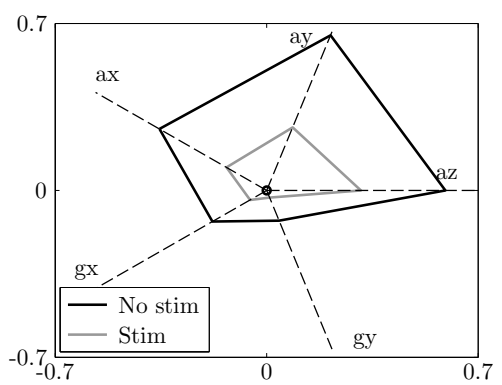

(i) Patient I

Figure 1: Tremor amplitude (RMS value) for all participating subjects measured at each sensor. $a$ refers to accelerometer
and $g$ to gyrometer. RMS values are shown in $\mathrm{m} / \mathrm{s}^{2}$ and $\mathrm{rad} / \mathrm{s}$, respectively. The axes limits are not the same for all patients, reflecting the different levels of tremor severity. 


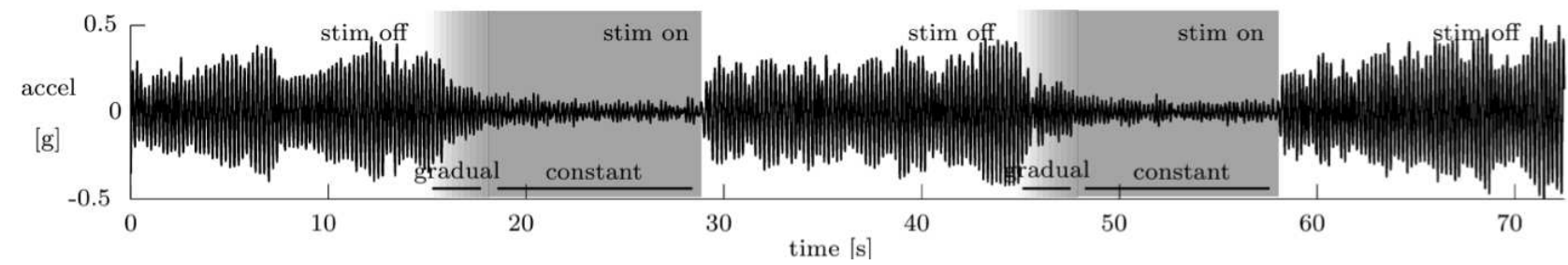

(a) Patient F

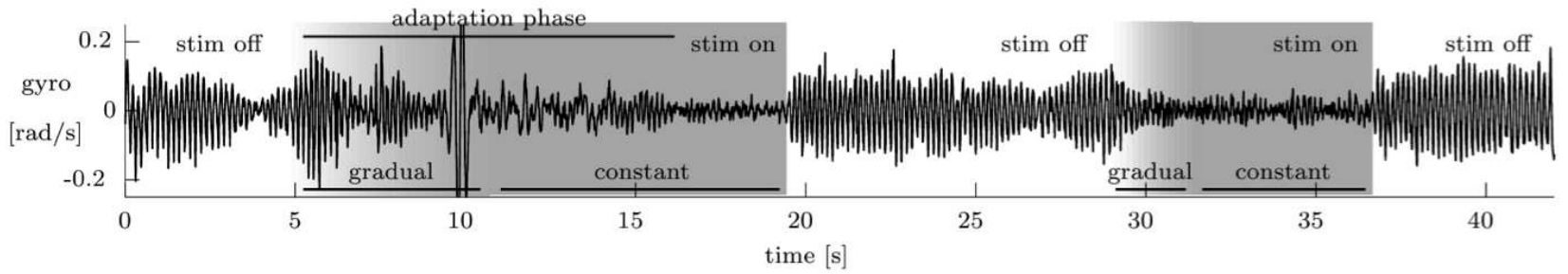

(b) Patient E

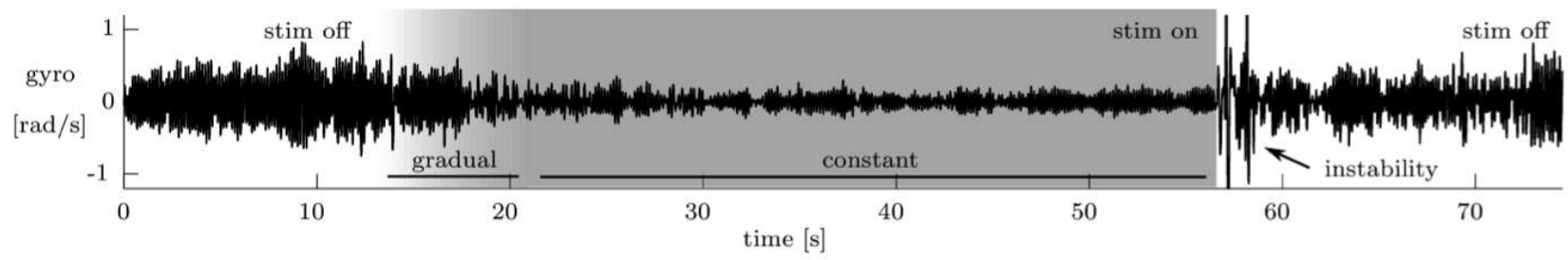

(c) Patient $\mathrm{H}$

Figure 2: Motion signals from illustrative trials. In (a), clear immediate attenuation may be observed. The adaptation phase required to achieve better performance is depicted in (b). In (c), the sudden instability that occurs once the support from FES vanishes is illustrated. In all cases, the initial period when FES intensity is gradually increased is also depicted.

The general performance of the method for all patients is also illustrated in Fig. 1. Data from all sensors and the corresponding mean tremor amplitude (represented by its RMS value) for the period in which FES was on and off is shown. Since the fixed-level stimulation may displace the joint and affect the resulting plane of motion of tremor, evaluating all measured axes is important to verify if tremor has shifted to a different plane, which would thus prevent an effective functional benefit to the user.

Finally, in order to illustrate motion response with respect to time, pieces of data that contain representative effects to substantiate the Discussion are depicted in Fig. 2. The figure illustrates one case where clear immediate tremor attenuation was observed, as well as others where an adaptation phase was required and an extra instability emerged when the FES system was turned off. For each motion, the sensor presenting highest RMS value was chosen (accelerometer for Fig. 2(a) and gyrometer for Fig. 2(b) and 2(c)).

\section{DISCUSSION}

Based on the illustrated results, it may be concluded that tremor attenuation based on fixed-intensity FES is feasible, 
since in only 2 patients in a population of 10 a poor or limited performance was observed. In this section we present a qualitative discussion concerning the major related issues.

Clear immediate tremor attenuation was detected on patients presenting different tremor features (in terms of affected joint and tremor severity given by the Fahn-Tolosa-Marin tremor rating scale) and types of stimulation strategy (single muscle or pair of antagonist muscles). This may indicate that separate phenomena are involved in the reduction of tremor amplitude, such as FES-induced co-contraction to increase joint impedance, reduction in tremor activity due to recruitment of muscle fibers presenting tremor activity or tremor attenuation due to unknown effects related to the stimulation of afferent pathways. Even though all these distinct hypotheses are plausible, based on the obtained data it is unrealistic to develop further conclusions due to the limited knowledge on ET muscle dynamics and the diversity between FES-induced muscle responses observed on different patients.

Concerning the circumstances where attenuation was preceded by an adaptation phase (e.g., Fig. 2(b)), we believe that the performance was greatly influenced by involuntary reactions to the FES-induced contraction. Since none of the patients had any previous experience with FES, the effect is unsurprising. For this reason, we believe patients that presented an adaptation phase before tremor attenuation may greatly benefit from adequate training. It may be observed within the referred figure, for instance, that there was no adaptation phase in the second stimulation period. Indeed, the adoption of stimulation trials featuring different intervals has enabled observation of higher tremor attenuation for longer trials in some patients. Other observed negative effects may be potentially reduced with such previous practice, such as the sudden instability generated once the support from FES is removed (e.g., Fig. 2(c)).

There were also 2 cases where no positive effect was observed during the experimental sessions, either due to tremor increase or no significant improvement. Considering that in just a few patients it was impossible to verify reduction in tremor amplitude using the proposed strategy, we believe that the lack of success in these sessions was caused by the limitations of the experimental protocol. For the other patients to whom the method has provided positive response, but considerable irregularity (as subjective judgment by the patient), it may also be the case that these experimental difficulties have affected the overall performance of the method.

Among the most significant weakness within the experimental protocol, one important difficulty was related to electrode placement in elderly patients, particularly when multiple muscles are stimulated (since stimulation diffusion increases considerably). Indeed, for most patients it is hard to imagine real-life applications without improved electrode technology, such as implantable or multi-pad electrodes, such as [Popovic-Bijelic et al., 2005].

Another significant issue is related to tremor inherent variability. Since the study was based on a within-subject experiment design where FES was the controlled variable, ideally all other aspects that affect the response should be 
kept constant. Indeed, intrinsic fluctuations of ET amplitude may greatly affect the computed tremor reduction. Employing static tasks reduced the variability in tremor amplitude, but in some case fluctuations remained intense due to subject-specific features, such as voluntary contractions. A longer protocol involving multiple sessions would enable the patient to get used to FES, which was not possible in our study, since it was based on single sessions with each patient.

In view of those experimental limitations, our future work focuses on trials on patients based on multiple experimental sessions including improvements on the choice of muscle(s) to stimulate, as well as proper evaluation of the functional benefit provided by the method. A longer protocol, including an initial training period, may demonstrate if the negative effects observed in some patients may be suppressed. Finally, based on recorded motion and patient evaluation of fatigue and sensitivity, the following goal is to compare different compensation strategies in controlled scenarios.

Nevertheless, the proposed method has indeed demonstrated the feasibility of reducing tremor amplitude by applying fixed-level FES to the muscles acting on the concerned joint. Comparing with alternative systems, such as the anti-phase approach, the proposed method may indeed present some advantages. Firstly, the applied stimulation may be more comfortable and predictable, possibly increasing the acceptance ratio among potential users. The resulting device may also be more compact and simpler, requiring less sophisticated and cheaper hardware. Considering that tremor often propagates from proximal to distal joints, this may also be an important feature. Indeed, in anti-phase FES tremor compensation, unstable performance on proximal joints may increase tremor at the hand, while in the proposed method the effect of stimulation is intrinsically stabilizing. Furthermore, the results have also shown that tremor was not shifted to another plane of motion, which would indicate that the pathological motion was not actually compensated and consequently a great disadvantage of the method. Finally, an additional important result concerns the fact that not always clear immediate tremor reduction occurs. Indeed, due to the observed phenomena of adaptation periods to previous to tremor attenuation and sudden instability after stimulation, we infer that tremor compensation strategies based on FES should mandatorily include a training phase when the patient will be able to learn the motor patterns to use during stimulation.

\section{ACKNOWLEDGEMENTS}

Neuromedics and the Agence Nationale de la Recherce, in the context of the TREMOR Project, supported this work.

\section{REFERENCES}


[Bó and Poignet, 2010] A. P. L. Bó and P. Poignet. Tremor attenuation using FES-based joint stiffness control. In 2010 IEEE International Conference on Robotics and Automation (ICRA 2010), pages 2928 - 2933, 2010.

[Bó et al., 2011] A. P. L. Bó, P. Poignet, and C. Geny. Pathological tremor and voluntary motion modeling and online estimation for active compensation. IEEE Transactions on Neural Systems and Rehabilitation Engineering, 19(2):177-185, 2011.

[Bó et al., 2009] A. P. L. Bó, P. Poignet, D. Zhang, and W. T. Ang. FES-controlled co-contraction strategies for pathological tremor compensation. In 2009 IEEE/RSJ International Conference on Intelligent Robots and Systems (IROS 2009), pages 1633-1638, 2009.

[Gallego et al., 2013] J. A. Gallego, E. Rocon, J. M. Belda-Lois, and J. L. Pons. A neuroprosthesis for tremor management through the control of muscle co-contraction. Journal of NeuroEngineering and Rehabilitation, 10(36), 2013.

[Grimaldi and Manto, 2008] G. Grimaldi and M. Manto. Tremor: From Pathogenesis to Treatment. Mongan \& Claypool, 2008.

[Lynch and Popovic, 2008] C. L. Lynch and M. R. Popovic. Functional electrical stimulation. IEEE Control Systems Magazine, 28(2):40-50, April 2008.

[Lyons and Pahwa, 2005] K. E. Lyons and R. Pahwa. Handbook of Essential Tremor and Other Tremor Disorders. Taylor \& Francis, 2005.

[Maneski et al., 2011] L. P. Maneski, N. Jorgovanovic, V. Ilic, S. Dosen, T. Keller, M. B. Popovic, and D. B. Popovic. Electrical stimulation for the suppression of pathological tremor. Medical and Biological Engineering and Computing, 49(10):1187-93, 2011.

[Popovic and Sinkjaer, 2000] D. B. Popovic and T. Sinkjaer. Control of Movement for the Physically Disabled: Control for the Rehabilitation Technology. Springer-Verlag, 2000.

[Popovic-Bijelic et al., 2005] A. Popovic-Bijelic, G. Bijelic, N. Jorgovanovic, D. Bojanic, M. B. Popovic, and D. B. Popovic. Multi-field surface electrode for selective electrical stimulation. Artificial Organs, 29(6):448-452, June 2005.

[Prochazka et al., 1992] A. Prochazka, J. Elek, and M. Javidan. Attenuation of pathological tremors by functional electrical stimulation I: Method. Annals of Biomedical Engineering, 20(2):205-224, 1992.

[Rocon et al., 2007] E. Rocon, J. M. Belda-Lois, A. F. Ruiz, M. Manto, J. C. Moreno, and J. L. Pons. Design and validation of a rehabilitation robotic exoskeleton for tremor assessment and suppression. IEEE Transactions on Neural Systems and Rehabilitation Engineering, 15(3):367-378, September 2007.

[Winters and Crago, 2000] J. M. Winters and P. E. Crago. Biomechanics and Neural Control of Posture and Movement. Springer-Verlag, 2000.

[Zhang et al., 2009] D. Zhang, P. Poignet, A. P. L. Bó, and W.T. Ang. Exploring peripheral mechanism of tremor on neuromusculoskeletal model: A general simulation study. IEEE Transactions on Biomedical Engineering, 56(10):2359-2369, 2009.

[Zhang et al., 2011] D. Zhang, P. Poignet, F. Widjaja, and W. T. Ang. Neural oscillator based control for pathological tremor suppression via functional electrical stimulation. Control Engineering Practice, 19(1):74-88, 2011. 